Pernille Juhl ${ }^{1}$, Anne-Christine Bay-Jensen ${ }^{2}$, Roger Hesselstrand ${ }^{3}$, Anne Sofie Siebuhr ${ }^{2}$, Dirk Wuttge ${ }^{3} .{ }^{1}$ University of Copenhagen, Department of Biomedical Sciences, Copenhagen, Denmark, ${ }^{2}$ Nordic Bioscience, Biomarkers and Research, Herlev, Denmark; ${ }^{3}$ University of Lund, Department of Clinical Sciences, Lund, Denmark

Background: The hallmark of systemic sclerosis (SSc) is fibrosis of the skin. The interstitial matrix is rich in type I and III collagen while type IV collagen is the main collagen of the basement membrane. These two types of matrix are anchored together by other matrix proteins such as type VI collagen. Tissue turnover is in a delicate equilibrium of tissue formation and degradation, which may be altered in pathologies such as $\mathrm{SSc}$, in which there is a net increase in tissue. By using advanced serological biomarkers tissue turnover, tissue formation and tissue degradation may be assed separately, to quantify the tissue balance. The hypothesis of the current study was that the tissue balance was altered in SSc as compared to healthy, and that limited and diffuse SSc would present with different turnover rates.

Objectives: The objective was to quantify the tissue turnover balance in a cross-sectional study in limited and diffuse SSc.

Methods: Forty-three patients fulfilling the 2013 ACR/EULAR criteria for SSc were included. The study included limited (ICSSc, $n=20$ ) and diffuse SSc (dcSSc, $n=23$ ) (recruited at Lund University, approval number Dnr 590/2008). Ten healthy controls were included.

Biomarkers of type III, IV and VI collagen formation (PRO-C3, PRO-C4, PRO-C6) and degradation (C3M, C4M, C6M) were measured cross sectional in serum samples by competitive ELISAs. The fibrotic index of collagens (FICol) were examine (formation/degradation). Difference between groups were tested by Mann-Whitney $U$ test and correlations by Spearman's correlation.

Results: There was no significant difference in gender, age or BMl between IcSSc and dSSc. The mean age of the population was 53.5 years and $79 \%$ were female. LcSSc had a longer disease duration (IcSSc: 32.3 month (SD: 41.0), dcSSc: 13.5 month (SD 14.9), $\mathrm{P}=0.05$ ) and a lower modified Rodnan skin score (mRSS, IcSSc: 4.25 points (SD: 3.1 ), dcSSc: 20.3 points (11.7), $\mathrm{P}<0.0001)$ compared to dcSSc as expected. Type III and VI collagen formation were both significantly increased in dcSSc compared to IcSSc (PRO-C3: $18.6 \mathrm{ng} / \mathrm{ml}$ (SD: 10.0) vs $13.0 \mathrm{ng} / \mathrm{ml}$ (SD: 6.5) $(P=0.01)$, PRO-C6: $16.7 \mathrm{ng} / \mathrm{ml}$ (SD: 6.6) vs $12.1 \mathrm{ng} / \mathrm{ml}$ (SD: 4.9) $(P=0.01)$, Figure 1). Type III collagen degradation were decreased in dcSSc compared to IcSSc (C3M: $13.3 \mathrm{ng} / \mathrm{ml}$ (SD: 2.9) vs $17.4 \mathrm{ng} / \mathrm{ml}$ (SD: 7.5) $(P=0.03)$ ). Neither type IV collagen formation nor type III, IV or $\mathrm{VI}$ collagen degradation were significantly different. The fibrotic index of type III and VI collagen (FICol3 and FICol6) were significantly increased in dcSSc compared to IcSSc (FICol3: 1.4 (SD: 0.6) vs 0.8 (SD: 0.3) $(\mathrm{P}=0.0001)$, FICol6: 1.2 (SD: 0.5$)$ vs 0.9 (SD: 0.3$)(P=0.03)$, Figure 2). The fibrotic index of type IV collagen (FICol4) were not different between the two groups but were both 1.5 times higher than the levels of healthy (healthy: 6.9, IcSSc 10.4 (SD: 3.3), dcSSc: 10.5 (SD:3,1)).

Both type III and VI collagen formation correlated moderately with mRSS with rho's of $0.53(P=0.0003)$ and $0.4(p=0.008)$, respectively. FICol3 correlated with a rho of $0.59 \quad(\mathrm{P}<0.0001)$ and FICol6with a rho of 0.35 $(P=0.04)$.
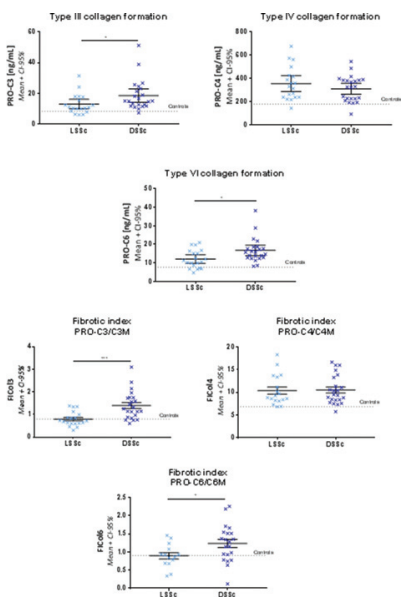

Conclusion: The tissue turnover balance was clearly altered in both limited and diffuse SSc as compared to healthy controls. In addition, diffuse

SSc presented with a more screwed balance in the fibrotic index towards tissue formation and disease progression. This study shows the importance of looking at both collagen formation and degradation. The collagen tissue turnover could be beneficial in following patients' fibrosis development and possibly identifying if they are progressing or in remission.

Disclosure of Interests: Pernille Juhl: None declared, Anne-Christine BayJensen Shareholder of: I own shares of Nordic Bioscience, Employee of: I am a full-time employee in Nordic Bioscience, Roger Hesselstrand: None declared, Anne Sofie Siebuhr Employee of: I am a full-time employee in Nordic Bioscience, Dirk Wuttge: None declared DOI: 10.1136/annrheumdis-2019-eular.5648

\section{FRI0327 EVALUATION OF AMERICAN COLLEGE OF RHEUMATOLOGY PROVISIONAL COMPOSITE RESPONSE INDEX IN SYSTEMIC SCLEROSIS IN A PHASE II TRIAL OF ABATACEPT VS. PLACEBO}

Dinesh Khanna, Cathie Spino, ASSET Investigators. University of Michigan, Ann Arbor, United States of America

Background: Treatment with CTLA4lg, abatacept (ABA), in early diffuse systemic sclerosis (dcSSc; a Phase 2 ASSET trial) showed evidence of improvements in modified Rodnan skin score (mRSS) and secondary outcome measures at month 121; however, statistical significance was not always shown vs. placebo. The ACR CRISS index, a composite outcome measure for trials in $S_{S c^{2}}$, is a 2-step process that assigns a probability of improvement for each subject ranging from 0 [no improvement] to 1 [marked improvement]. Step 1 assesses clinically meaningful decline in cardio-pulmonary-renal involvement with a probability of 0 . For remaining subjects, probability of improvement is based on 5 variables: changes from baseline to month 12 in FVC\%, mRSS, patient (PTGA) and physician global assessments (MDGA), and HAQ-DI.

Objectives: We assessed the performance of ACR CRISS, a secondary outcome measure, in ASSET at month12.

Methods: ASSET was a multicenter double-blind, randomized placebo-controlled trial. Eligible subjects were randomized in a $1: 1$ ratio to either 12 months of $125 \mathrm{mg} \mathrm{SC} \mathrm{ABA}$ or matching placebo, stratified by duration of dcSSc ( $\leq 18$ vs $>18$ to $\leq 36$ months). Investigators reported SSc end organ involvement (Step 1 for ACR CRISS) prospectively using a case report form. All AEs and SAEs were reviewed for cardio-pulmonary and renal involvement by the study PI. Step 2 calculated the ACR CRISS index as previously defined. Treatment differences, adjusted for duration of dcSSc, in the CRISS score were assessed by the non-parametric Van Elteren test and by ANCOVA for individual CRISS components. We calculated Spearman's correlation coefficients to assess the relationship between the ACR CRISS score and individual components. Multiple imputation was used for analysis, creating 25 complete datasets with estimates, standard errors and p-values pooled over each imputed dataset.

Results: 88 subjects (44 ABA, 44 PBO) were randomized; 63 (72\%) had complete data for all relevant outcomes at month 12. 5 PBO and 5 ABA subjects met the pre-defined definition of worsening cardio-pulmonary-renal involvement (Step 1) and were given a score of 0 . There is evidence of improved ACR CRISS scores on ABA compared to the PBO at month 12 and the difference was statistically significant $(p=0.03$; Table). Most variables, except HAQ-DI and PTGA, had statistically significant correlations with the ACR CRISS (Table).

Conclusion: In ASSET trial, the ACR CRISS was more sensitive to clinically meaningful treatment changes than the skin score and provides further validation of ACR CRISS as an independent primary endpoint for clinical trials.

\section{REFERENCES:}

[1] Khanna D. ACR Presentation 2018

[2] Khanna D. Arthritis Rheumatol. 2016

Acknowledgement: This project was supported by NIH/NIAID Clinical ACE grant (5UM1Al110557-05) and an investigator-initiated grant by Bristol-Myers Squibb

Disclosure of Interests: Dinesh Khanna Shareholder of: Eicos Sciences, Inc, Grant/research support from: Bayer, BMS, Pfizer, Horizon, Consultant for: Actelion Acceleron, Arena, Bayer, BI, BMS, CSL Behring, Corbus, Cytori, GSK, Genentech/Roche, Galapagos, Employee of: Elcos Sciences, Inc, Cathie Spino: None declared DOI: 10.1136/annrheumdis-2019-eular.1714 
Table. Spearman Correlations between CRISS and individual components at 12 months and Comparison of ABA and PBO using CRISS index and individual components at 12 months;

\begin{tabular}{|c|c|c|c|c|c|}
\hline Outcome & & $\begin{array}{l}\mathrm{ABA} \\
\mathrm{N}=44\end{array}$ & $\begin{array}{l}\mathrm{PBO} \\
\mathrm{N}=44\end{array}$ & $\begin{array}{c}\text { Treatment } \\
\text { Difference (ABA- } \\
\text { PBO) }\end{array}$ & $\begin{array}{c}\mathrm{P}- \\
\text { value }^{\wedge}\end{array}$ \\
\hline \multirow[t]{2}{*}{$\begin{array}{l}\text { ACR CRISS (0.0-1.0) } \\
\text { median (IQR) }\end{array}$} & & $\begin{array}{c}0.68 \\
(1.00)\end{array}$ & $\begin{array}{c}0.01 \\
(0.86)\end{array}$ & & 0.03 \\
\hline & $\begin{array}{l}\text { Spearman } \\
\text { Correlation }\end{array}$ & $\begin{array}{l}\text { LS } \\
\text { mean } \\
(\mathrm{SE})\end{array}$ & $\begin{array}{l}\text { LS } \\
\text { mean } \\
(\mathrm{SE})\end{array}$ & $\begin{array}{l}\text { LS mean } \\
\quad(\mathrm{SE})\end{array}$ & $\begin{array}{c}\mathrm{P}- \\
\text { value }^{\wedge \wedge}\end{array}$ \\
\hline$\Delta \mathrm{mRSS}(0-51)$ & $-0.75^{\star}$ & $\begin{array}{c}-6.7 \\
(1.30)\end{array}$ & $\begin{array}{c}-3.8 \\
(1.23)\end{array}$ & $-2.9(1.75)$ & 0.10 \\
\hline$\triangle \mathrm{FVC} \%$ predicted & $0.36^{*}$ & $\begin{array}{c}-1.4 \\
(1.30)\end{array}$ & $\begin{array}{c}-3.1 \\
(1.20)\end{array}$ & $1.7(1.72)$ & 0.32 \\
\hline$\triangle$ PTGA $(0-10)$ & -0.17 & $\begin{array}{c}-0.50 \\
(0.392)\end{array}$ & $\begin{array}{l}-0.30 \\
(0.385)\end{array}$ & $-0.20(0.557)$ & 0.73 \\
\hline$\triangle \mathrm{MDGA}(0-10)$ & $-0.47^{*}$ & $\begin{array}{c}-1.34 \\
(0.282)\end{array}$ & $\begin{array}{l}-0.18 \\
(0.284)\end{array}$ & $-1.16(0.403)$ & 0.004 \\
\hline$\triangle \mathrm{HAQ}-\mathrm{DI}(0-3)$ & -0.21 & $\begin{array}{c}-0.11 \\
(0.079)\end{array}$ & $\begin{array}{c}0.11 \\
(0.076)\end{array}$ & $-0.22(0.108)$ & 0.05 \\
\hline
\end{tabular}

${ }^{\wedge} \mathrm{p}$-value for treatment comparisons based on Van Elteren test ${ }^{\wedge} \mathrm{p}$-value for treatment comparisons based on ANCOVA model with treatment, duration of SSc and baseline value as covariates ${ }^{*} p<0.01$ using Spearman correlation coefficientNegative score denotes improvement, except for FVC\% where negative score denotes worsening; LS mean = least squares mean; $\mathrm{SE}=$ standard error

\section{FRI0328 BRANCHED CHAIN AMINO ACIDSIN THE TREATMENT OF POLYMYOSITIS AND DERMATOMYOSITIS: RESULTS FROM THE BTOUGH STUDY}

Naoki Kimura, Hitoshi Kohsaka. Tokyo Medical and Dental University (TMDU), Department of Rheumatology, Tokyo, Japan

Background: Muscle functions of patients with polymyositis and dermatomyositis (PM/DM) remain often impaired even after successful control of the immune-mediated muscle injury by immunosuppressive therapy. The only effort at the present to regain muscle functions except for the immunosuppression is rehabilitation, which is carried out systematically in limited institutes. No medicines for rebuilding muscles have been approved. Branched chain amino acids (BCAA) promote skeletal muscle protein synthesis and inhibit muscle atrophy. They thus have positive effects on muscle power, but have never been examined for the effects on PM/DM patients.

Objectives: To assess the efficacy and safety of BCAA in the treatment of PM/DM for official approval of their use in Japan.

Methods: Untreated adults with PM/DM were enrolled in a randomized, double-blind trial to receive either TK-98 (drug name of BCAA) or placebo in addition to the conventional immunosuppressive agents. One package of TK-98 $(4.15 \mathrm{~g})$ contained L-isoleucine 952mg, L-leucine $1904 \mathrm{mg}$, and L-valine $1144 \mathrm{mg}$ (molar ratio is 1:2:1.35), and 6 packages were administered daily in 3 divided doses. After 12 weeks, patients with average manual muscle test (MMT) score less than 9.5 were enrolled in an open label extension study for 12 weeks. The primary end point was the change of the MMT score at 12 weeks. The secondary end points were the disease activity evaluated with myositis disease activity core set (MDACS) and the change of functional index (FI), which evaluates dynamic repetitive muscle functions.

Results: Forty-seven patients were randomized to the TK-98 (24 patients [12 with PM and 12 with DM]) and placebo (23 patients [11 with PM and 12 with DM]) groups. The baseline MMT scores were equivalent $(7.97 \pm 0.92$ [mean $\pm S D$ ] in the TK-98 group and $7.84 \pm 0.86$ in the placebo group). The change of MMT scores at 12 weeks were $0.70 \pm 0.19$ (mean \pm SEM) and $0.69 \pm 0.18$, respectively $(P=0.98)$. Thirteen patients from the TK-98 group and 12 from the placebo group were enrolled in the extension study. The MMT scores in both groups improved comparably throughout the extension study. The increase of the $\mathrm{FI}$ scores of the shoulder flexion at 12 weeks was significantly larger in the TK-98 group $(27.9 \pm 5.67$ and $12.8 \pm 5.67$ in the right shoulder flexion $[P<0.05], 27.0$ \pm 5.44 and $13.4 \pm 5.95$ in the left shoulder flexion $[P<0.05]$ ). The improvement rate of the average $\mathrm{FI}$ scores of all tested motions (head lift, shoulder flexion, and hip flexion) through the first 12 weeks was larger in the TK-98 group. No difference was found in the disease activity throughout the study period. Frequencies of the adverse events until 12 weeks were comparable.
Conclusion: Although BCAA exerted no effects in the improvement of the muscle strength evaluated with MMT, they were effective in the improvement of dynamic repetitive muscle functions in patients with PM/DM with out significant increase of adverse events.

Disclosure of Interests: None declared

DOI: 10.1136/annrheumdis-2019-eular.5235

\section{FRI0329 ANALYSIS OF 11 CASES OF ANTI-PL-7 ANTIBODY POSITIVE PATIENTS WITH IDIOPATHIC INFLAMMATORY MYOPATHIES. MALIGNANCY MAY NOT BE UNCOMMON COMPLICATION IN ANTI-PL-7 ANTIBODY POSITIVE MYOSITIS PATIENTS}

Taiga Kuga, Yoshiyuki Abe, Masakazu Matsushita, Kurisu Tada, Ken Yamaji, Naoto Tamura. Juntendo University School of Medicine, Department of Internal Medicine and Rheumatology, Tokyo, Japan

Background: Various autoantibodies are known to be related to idiopathic inflammatory myopathies (IIM). Anti-PL-7 antibody is anti-threonyl-tRNA synthetase antibody associated with antisynthetase syndrome (ASS). Since anti-PL-7 antibody is rare (mostly $1-4 \%$ of myositis, while a Japanese study reported 17\%), little is known as to clinical characteristics of it (1). Objectives: To analyze clinical characteristics of anti-PL-7 positive IIM patients.

Methods: Anti-PL-7 antibody was detected by EUROLINE Myositis Profile 3. IIM diagnosis was made by the 2017 EULAR/ACR classification criteria (2) and/or Bohan And Peter classification (3). Eleven anti-PL-7 antibody positive adult patients (all female), age at onset (61.5 \pm 12.6 years) were enrolled in this study between 2009 and 2018. Clinical manifestations, laboratory and instrumental data were reviewed in this single centre retrospective cohort.

Results: Characteristic symptoms were identified at diagnosis: skin manifestations (7/11 cases, $63.6 \%)$, muscle weakness (8/11 cases, $72.7 \%)$, arthralgia (5/11 cases, 45.5\%) and Raynaud's phenomenon (4/11 cases $36.4 \%$ ). Myogenic enzymes were elevated in most cases (10/11 cases, $90.9 \%$ ). ILD was detected in all patients (11/11 cases, $100 \%$ ) and 2 patients (18.2\%) developed rapidly progressive ILD. Largest IIM subtype was polymyositis (PM, 5/11 cases), followed by dermatomyositis (DM, 3/ 11 cases) and amyopathic dermatomyositis (ADM, 3/11 cases). Five patients $(45.5 \%)$ complicated with malignancy within 3 years from the diagnosis of IIM. Though clinical manifestations and laboratory data showed any difference between malignancy group and non-malignancy group, all 3 ADM cases but no DM cases complicated with malignancy in this study.

Conclusion: Anti-PL-7 antibody positive IIM patients frequently complicated with ILD. Frequency of cancer in ASS patients within three years from diagnosis was $1.7 \%$ and not much different from the general population in previous report from France (4). Though this study only included IIM patients and may have selection bias, careful malignancy survey may be essential in Anti-PL-7 antibody positive IIM patients.

\section{REFERENCES}

[1] Y Yamazaki, et al. Unusually High Frequency of Autoantibodies to PL-7 Associated With Milder Muscle Disease in Japanese Patients With Polymyositis/DermatomyositisARTHRITIS \& RHEUMATISM Vol. 54, No. 6 , June 2006, pp 2004-2009

[2] Lundberg IE, Tjärnlund $A$, Bottai $M$, et al. EULAR/ACR classification criteria for adult and juvenile idiopathic inflammatory myopathies and their Major Subgroups. Ann Rheum Dis. 2017;76:1955-64.

[3] Bohan A, Peter J. Polymyositis and dermatomyositis. N Engl J Med 1975, 292: 344-347; 403-407.

[4] Hervier B, et al. Hierarchical cluster and survival analyses of antisynthetase syndrome: phenotype and outcome are correlated with anti-tRNA synthetase antibody specificity. Autoimmunity reviews. 2012; 12:210-217.

Disclosure of Interests: Taiga Kuga: None declared, Yoshiyuki Abe: None declared, Masakazu Matsushita: None declared, Kurisu Tada Grant research support from: Eli Lilly, Ken Yamaji: None declared, Naoto Tamura Grant/research support from: Astellas Pharma Inc., Asahi Kasei Pharma, AYUMI Pharmaceutical Co., Chugai Pharmaceutical Co. LTD, Eisai Inc., :Takeda Pharmaceutical Company Ltd., Speakers bureau: Jans sen Pharmaceutical K.K., Bristol-Myers Squibb K.K., :Mitsubishi Tanabe Pharma Co.

DOI: 10.1136/annrheumdis-2019-eular.4150 